\title{
The Strategy of Ontology-based Semantic Replica in Cloud Storage
}

\author{
Wang Shunyan, Zhou Haitao, Wang Jun, Cao Fangfang \\ School of Computer Science and Technology \\ Wuhan University of Technology \\ Wuhan,China \\ e-mail:wangsyan@whut.edu.cn;zht_whut@sina.cn
}

\begin{abstract}
With the development of cloud computing, cloud storage distributed the endless file system,but not a better copy strategy compatible with various file systems.This paper proposed ontology-based metadata replica which make different file systems mutual fusion ,and give a policy of replica allocation of low-load access .
\end{abstract}

Keywords- Cloud Storage; Ontology semantic; mutual fusion

\section{INTRODUCTION}

With the rapid development of the cloud computing technology ${ }^{[1,2]}$, mass data storage has increasingly become the problems and challenges faced by many data-intensive enterprise, including structured,semi-structured and unstructured information stored. To this end the major companies competing a relatively suitable solution, but not one for the future of information. In this article, the strategy of ontology-based semantic replica is based on the future information which may arise, through classifying storage systems with semantic and then make the metadata of the corresponding semantic routing to the appropriate storage system.

\section{RELATED RESEARCH}

\section{A. Status of commonly-used distributed file system}

Cloud storage is based on the Internet globally distributed resources to store and access models, high transmission rate and high throughput data services can be provided to a large number of users at the same time.Cloud storage is an important foundation for cloud computing and the main application field, able to manage large data sets, efficient massive data storage, processing, analysis and access to specific objects.

Existing distributed storage technology, including Google Bigtable ${ }^{[3]}$, HDFS ${ }^{[4]}$, Taobao FS ${ }^{[5]}$, Tecent FS, Amazon Dynamo ${ }^{[6]}$,Amazon Simple Storage Service (S3) and Facebook Haystack. Bigtable is the representative of the central system, Google used to solve the massive amounts of structured data. HDFS is used for the massive large data, Taobao FS, Tecent FS and Haystack, respectively, for the massive picture data in Taobao, Tencent (China) and Facebook

Dynamo and S3 Amazon company for Internet data and Dynamo representatives of peer-to-peer system. Most of the above system has a similar design:A Master machine plus multiple machine architecture, Master Slave machine storage system all metadata and data distribution, replication, backup, decision-making,and metadata checkpoint and operation logging and playback functions. Slave machine storage data

And data storage, data migration and data calculated in accordance with the instructions of the Master machine.

But these distributed storage system has the following three disadvantages:

1)There is no uniform application layer API interface, the lack of good compatibility.

2)Suited to deal with only a a relatively single type of data, such as HDFS for processing large data and frequently read data and not suitable for small files and frequently write data; Taobao FS is more suitable for the small picture type file.

3)The metadata of a single node.

\section{B. Commonly-used strategy of metadata replica management}

Replica management strategy is often used in large-scale cloud storage systems, to ensure the system's high reliability,high availability and efficiency of the system requirements, the copy of the policy management usually includes a copy of redundancy and replica consistency. The number of replica of redundant HDFS and Amazon Dynamo is 2 , and this number is usually adjusted accordingly do with the frequency of user access, access frequency increases with the increase of the number of copies and are not necessarily linked. Literature [7] of the study showed that the user data access having the burst, but part of the burst access request maintenance time is very short, in this case, if the burst access request to believe that the excessive heat of the file and create a new replica, but will spend a lot of overhead, reduce system performance. Therefore, the file current short time visits to measure heat will cause unnecessary replica creation. Literature [8] replica of redundancy management strategy based on the value of the user experience. Literature 9 in the grid environment with a copy of the data strategy using dynamic voting mechanism. Replica consistency is generally based on the consistency of the hash algorithm to achieve eventual consistency of the data, this part does not discuss in this article. 


\section{THE STRATEGY OF ONTOLOGY-BASED SEMANTIC REPLICA}

\section{A. Ontology semantic classification}

In the computer field, Gubrer given the body of one of the most popular definition :An of ontology is an explicit specification of a conceptualization (ontology is a clear specification of the conceptual model) ${ }^{[10]}$. The body can be divided into two categories, a class ontology ontology class features such service ontology classification according to their hierarchical relationships organization; another type of body is a special case, with the exception body may be a class, but also may be only one individual.The Ontology schematic as follow:

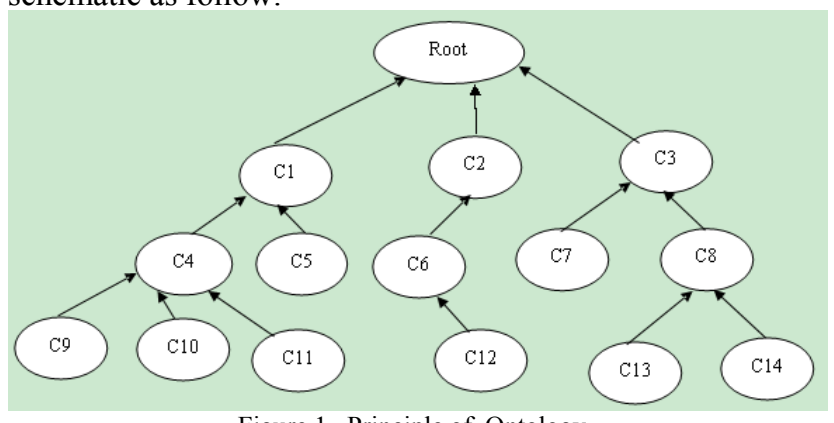

Figure 1. Principle of Ontology

The tasks of the classification system of the replica is given under the classification system, according to the content and properties of the meta-data to automatically determine the associated category. From a mathematical point of view, replica classification is a mapping process, will not indicate the category of metadata is mapped to the existing categories to distribute copies of the purpose. The mapping is expressed mathematically as follows:

$$
\mathrm{f}: \mathrm{A} \rightarrow \mathrm{B} \text { (1) }
$$

Where in, A is to be classified metadata collection, $\mathrm{B}$ is a set of classes in the classification system, $\mathrm{f}$ is a function, or a model to create the A and B mapped. Replica classification mapping rules have mastered a number of samples of each type of data, the system summarizes the classification regularity establish the discriminant formula and discriminant rules and in the face of a new metadata based on the discrimination rules to determine metadata related to the category.

\section{B. Ontology Language}

The ontological semantics emphasis on the significance of the treatment without parsing, at least not primarily by parsing. Acceptance of the machine on the significance, characterization, processing, generate and output, not primarily rely on original keyword matching, syntax conversion, and rely on the full range of simulation of human intelligence. In engineering practice, the ontological semantics concerned about the processing of the meaning of the various factors, not only noted that intellectual factors, but also attach importance to the non-knowledge of factors underlying human intelligence, and specific ways they internalized artificial intelligence system being
constructed.Ontology description language for the domain model allows the user to write a clear, formal concept description, it should meet the following requirements : the well-defined syntax (a well-defined syntax), well-defined semantics (a well-defined semantics),effective reasoning support (efficient reasoning support), full of expression capabilities (sufficient expressive power), the expression of convenience (convenience of expression).

\section{OWL}

The full name of OWL is the Web Ontology Language, which is a W3C recommended Semantic Web ontology language standard. It developed from a combination of some European and American research institutions description language DAML + OIL. Stack of W3C Ontology Language, OWL is the top, as shown below:

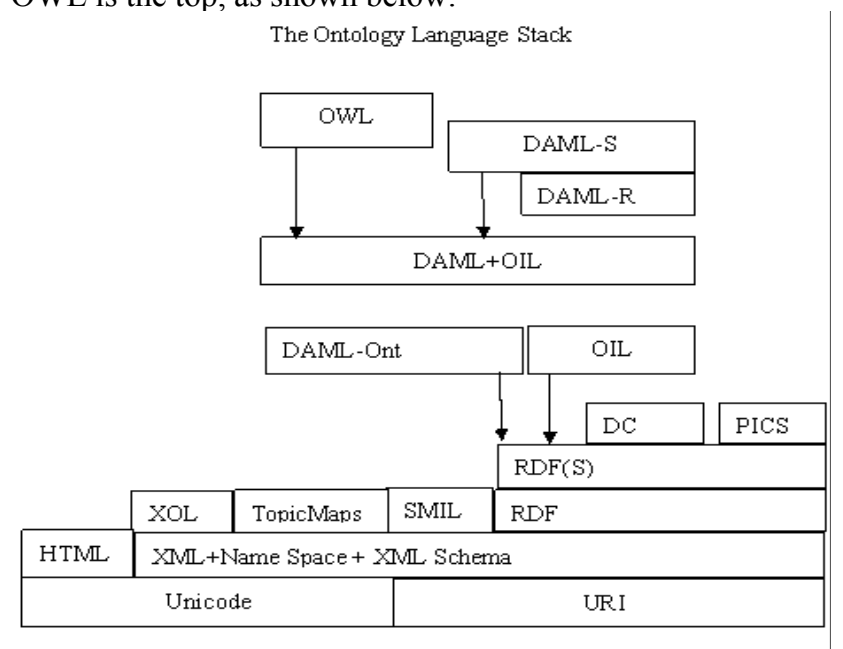

Figure 2. Ontology Language Architecture. For the different needs of OWL has three sub-language description of the list are as follows:

TABLE I. THREE OWL SUB-LANGUAGE

\begin{tabular}{|l|l|l|}
\hline Sub-langue & Description & Example \\
\hline OWL Lite & $\begin{array}{l}\text { Used to provide for those user who only need a } \\
\text { classification hierarchy and simple constraint } \\
\text { attributes }\end{array}$ & $\begin{array}{l}\text { Support cardinality , only for 0 } \\
\text { or 1 }\end{array}$ \\
\hline OWL DL & $\begin{array}{l}\text { Support for users who need the greatest degree } \\
\text { of expression based inference system, which } \\
\text { ensure that the the computational completeness } \\
\text { and decidability .Including all the constraints of } \\
\text { OWL, but only in specific constraints. }\end{array}$ & $\begin{array}{l}\text { When a class is a subclass of } \\
\text { multiple classes, it is } \\
\text { constrained not another } \\
\text { instance of the class }\end{array}$ \\
\hline OWL Full & $\begin{array}{l}\text { Support for users who need the RDF syntax } \\
\text { freedom guaranteed not calculated on the } \\
\text { maximum degree of expression. It allows to } \\
\text { increase predefined (RDF, OWL) vocabulary in } \\
\text { an ontology vocabulary, so that any reasoning } \\
\text { software can not support all the features of OWL } \\
\text { FULL. }\end{array}$ & $\begin{array}{l}\text { A class can be at the same time } \\
\text { expressed as a collection of } \\
\text { many individuals, and this }\end{array}$ \\
\hline
\end{tabular}




\section{Cloud STORAGE MOdEL BASED ON SEMANTIC DATA REPLICA MANAGEMENT STRATEGY}

\section{A. Improved cloud storage model}

According to SNIA agreement, cloud storage is divided into the four-layer model are the access layer, the application interface layer, infrastructure management, storage layer. Where the basis of management's cloud storage model core part, but also the most difficult to achieve. The basis of management through the cluster, distributed file systems and grid computing technology, collaborative work between multiple storage devices in the cloud storage, multiple storage devices can provide the same kind of service, and to provide a bigger and more powerful better data access performance. Distributed file system mentioned earlier, for different types of meta data, but not a unified interface between the file system can not be good fusion.

This ontology-based semantic replica, you can achieve the effect of integration between the two systems.A metadata belonging to a structured, semi-structured and unstructured, also can define the following semantics:

TABLE II.

SEMANTIC INFORMATION

\begin{tabular}{|l|l|l|l|l|l|l|l|l|}
\hline Size & $\begin{array}{l}\text { Only } \\
\text { Read }\end{array}$ & $\begin{array}{l}\text { Read } \\
\text {-Write }\end{array}$ & Operability & $\begin{array}{l}\text { Read } \\
\text { Frequency }\end{array}$ & $\begin{array}{l}\text { Write } \\
\text { frequency }\end{array}$ & $\begin{array}{l}\text { File } \\
\text { extensions }\end{array}$ & Time & Version \\
\hline
\end{tabular}

The the semantic metadata replica format as shown below:

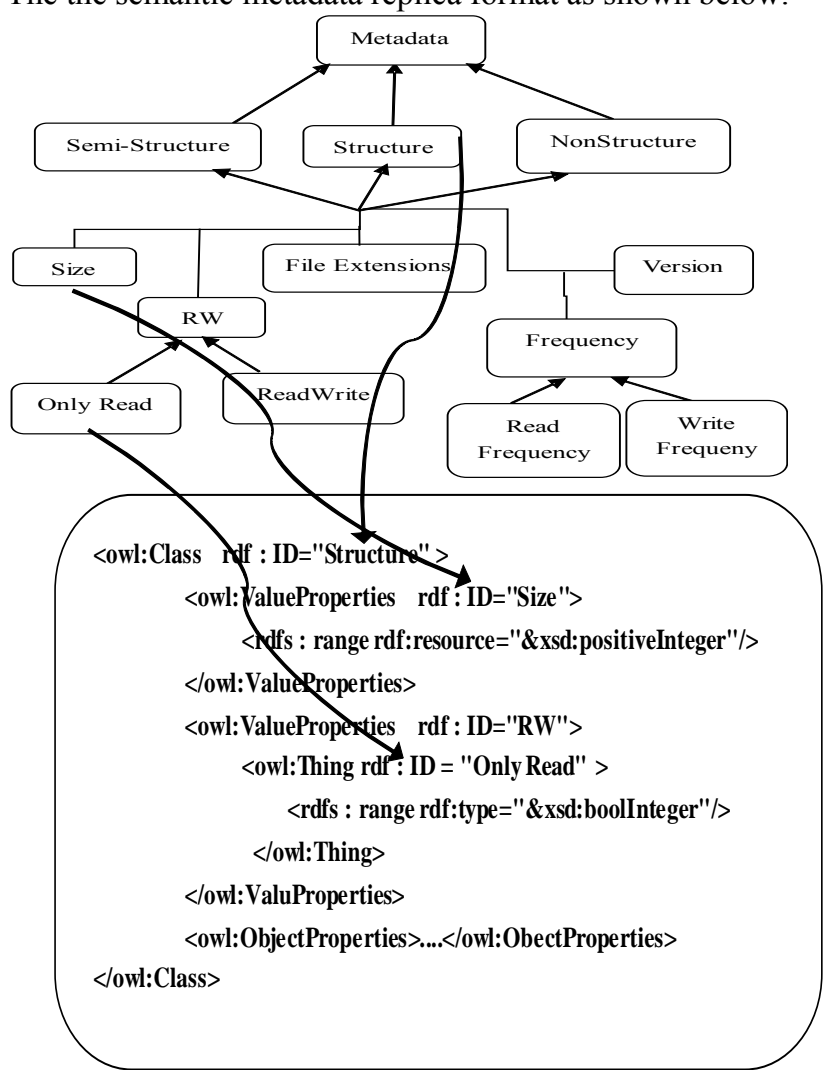

Figure 3. Using annotations to embed machine-processable metadata and extract formed definitions.
Metadata with adding semantic description of the different file system as a set of categories in the classification system. For each one needs the metadata stored by the replica classification system may be first, since meta-data using semantic description combined by the idea with the body, can determine the type of data distributed to each of different file systems, and then through a copy of the routing mechanism in. Therefore, the storage model as shown below:

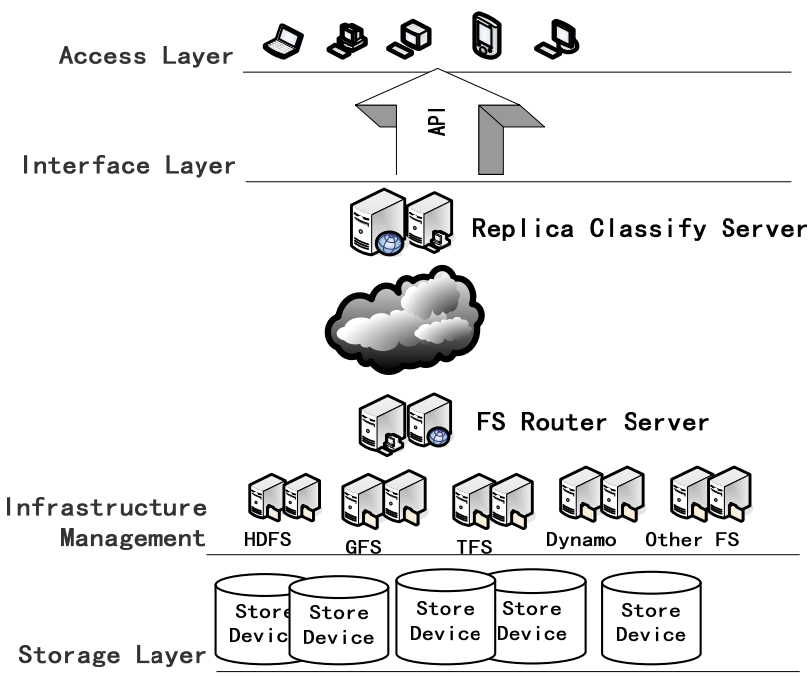

Figure 4. Cloud storage model.

\section{B. Improved metadata replica management strategy}

Description of the previous section, a copy of the body of the classification system based on semantic data mapping, and distributed to the appropriate type of file system, however, each distributed file system has a number of cluster nodes, routing controller when the file system metadata arrive after specific allocation where a node yet to be optimized. Traditional distributed file system, partial failure and data synchronization update overhead caused by the impact of system performance, so that the amount of data and the number of nodes restrictions ${ }^{[11]}$. Cloud storage is based on the Internet globally distributed resources to store and access models, high transmission rate and high throughput data services can be provided to a large number of users at the same time, you can dynamically determine the route with semantic metadata information the consideration to be distributed to different nodes.

File system routing controller is based on a multi-node, of these nodes denoted as $\mathrm{A}$, each node $\mathrm{AI} \in \mathrm{A}, \mathrm{i}=1,2, \ldots$, $\mathrm{m}$, the file system of each server node $\mathrm{B}, \mathrm{Bj}$ of $\in \mathrm{B} . \mathrm{j}=$ $1,2, \ldots, \mathrm{n}$, the node ai consideration for access to the server is recorded in the matrix: 


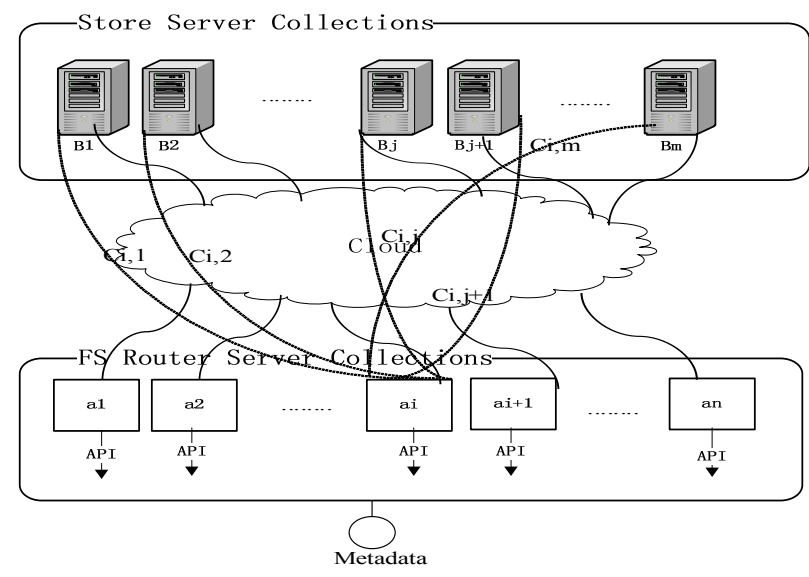

Figure 5. Replica Distribute Model in Infrastructure Management

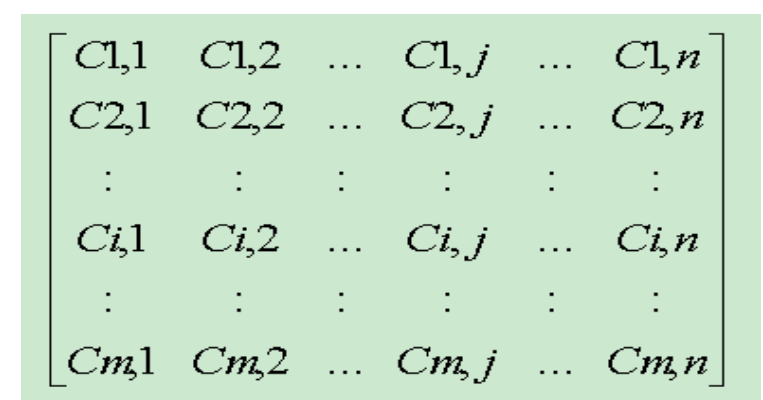

Where in: $\mathrm{Ci}, \mathrm{j}$ represents the service providing the point ai the storage server $B_{j}$ access consideration $(i=1,2, \ldots$, multiplet; $j=1,2, \ldots, n)$. Specific consideration of the access to the communication overhead, bandwidth allocation, hardware configuration, the storage capacity utilization and query load prime impact, its calculation see [12, 13].Found consideration of the minimum access two storage servers are arranged in a replica, which is equivalent to identify the m-th element in the matrix $\mathrm{C}$, this $\mathrm{m}$-th element belongs only to a matrix 2, and the element is the smallest. The application does not have access to the service delivery points involved in the calculation. Set $\mathrm{V}$ recording application point of access to the services provided:

$$
\mathrm{V}=\{\mathrm{v} 1, \mathrm{v} 2, \ldots, \mathrm{vm}\}(2)
$$

Where: if vi $=0$ indicates that the application is not in the service delivery points $i$ access data $r$;

if $v i=1$, then the application service provider point $i$ access data r. Unvisited node is not involved in the total consideration. Consider only part of the entire topology to reduce the complexity of the calculation,

calculation results and access consistent. Data $r$ by lowest price is calculated as follows:

$$
F_{\min -\cos t}(r, V)=\min _{1<<i, j, t<n}\left\{\sum_{k=1}^{m} V_{k} \times \min \{C k i, C k j, C k t\}\right\}
$$

Calculated by the formula (3) minimum access cost Fmincost, corresponding to $\mathrm{i}$ and $\mathrm{j}$ denote two copies should be stored in the storage server $\mathrm{Bi}, \mathrm{Bj}$ and $\mathrm{Bt}$.

\section{CONCLUSION}

Many involved in the research direction of the cloud storage, distributed storage system currently used by the cloud storage analysis, replica of the study, a strategy based on the the ontology semantics of data copies cloud storage model as well as a copy of allocation, the model is a good solution to the structured, semi-structured and structured mass data resources corresponding method of storing vast amounts of data on the different platforms for different categories of fusion solve different distributed storage consistency and operability of the system.

Specific research on cloud storage is also faced with many challenges, and through the body thinking on natural language processing there are many difficulties to be to solve the semantic replica put forward higher requirements for cloud storage, future study focus on the following aspects: 1) a specific implementation strategy of the semantic replica in the cloud storage system 2) semantic data copy consistency strategy research.

\section{REFERENCE}

[1] Hayes B. Cloud Computing [J].Communications of the ACM, 2008, 51(7):9-11.

[2] LIN G, DASMALCHI G, ZHU J. Cloud Computing and IT as a Service: Opportunities and Challenges [C].Proceedings of the IEEE 6th International Conference on Web Services (ICWS' 08), Sep 23-26, 2008, Beijing, China. Los Alamitos, CA,USA: IEEE Computer Society, 2008:5.

[3]Chang F, Dean J, Ghemawat S, et al. Bigtable: a distributed storage system for structured data[J]. ACM Transactions on Computer Systems, 2006, 26(2): 205-218.

[4]Borthakur D. HDFS architecture. Maryland,USA:The Apache Software Foundation,2008.[2011]. http://hadoop.apache.org/docs/r2.0.2-alpha/ .

[5]Taobao File System. 2011. http://code.taobao.org/p/tfs/wiki/intro/

[6]DeCandia G, Hastorun D, Jampani M, et al. Dynamo: amazon's highly available key-value store[C]// Proceedings of 21st ACM SIGOPS Symposium on Operating Systems Principles. Washington, USA: ACM, 2007: 205-220.

[7]Liu ya.Wireless sensor networks, energy model and simulation research[D]ChengDu,China: University of Electronic Science and Technology of China, 2008.

[8]Wang Q.X;Design and Implementation of replica with the user experience based on the value of the redundancy management system[D]ChengDu,China: University of Electronic Science and Technology of China, 2009.

[9]Wu,C.Z; Tian ,D; Wu,Z.F.The the dynamic voting mechanism data grid replica consistency maintenance [J]Computer Science.2009,Vol . 36 No. 4

[10] Gruber, T. A translation approach to portable ontologies. Knowledge 7. [11]Agrawal D, Abbadi A E, Antony Setal. Data management challenges in cloud computing infrastructures[C]//Proceedings of the 6th International Workshop on Databases in Networked Information Systems. Japan: Springer, 2010: 1-10.

[12] Bonvin N, Papaioannou T G, Aberer K. Dynamic cost-efficient replication in data clouds[C]// Proceedings of the 1st Workshop on Automated Control for Datacenters and Clouds. Barcelona, Spain: ACM, 2009: 49-56.

[13] Bonvin N, Papaioannou T G, Aberer K. A self-organized, faulttolerant and scalable replication scheme for cloud storage[C]// Proceedings of the 1st ACM Symposium on Cloud Computing. Indianapolis, Indiana, USA: ACM, 2010: 205-216. 